\title{
Cloud Service Matchmaking Approaches: A Systematic Literature Survey
}

\author{
Begüm İlke Zilci, Mathias Slawik, and Axel Küpper \\ Service-centric Networking \\ Technische Universität Berlin, Germany \\ \{ilke.zilci|mathias.slawik|axel.kuepper\}@tu-berlin.de
}

\begin{abstract}
Service matching concerns finding suitable services according to the service requester's requirements, which is a complex task due to the increasing number and diversity of cloud services available. Service matching is discussed in web services composition and user oriented service marketplaces contexts. The suggested approaches have different problem definitions and have to be examined closer in order to identify comparable results and to find out which approaches have built on the former ones. One of the most important use cases is service requesters with limited technical knowledge who need to compare services based on their QoS requirements in cloud service marketplaces. Our survey examines the service matching approaches in order to find out the relation between their context and their objectives. Moreover, it evaluates their applicability for the cloud service marketplaces context.
\end{abstract}

Keywords-Service matchmaking; Service matching; Cloud; Service marketplaces; Survey

\section{INTRODUCTION}

The increasing number and diversity of services available in the cloud computing environment causes the service selection to become more complex. Cloud service marketplaces in the industry provide merely keyword search and categories to select applications from such as marketing and customer management [1], [2]. Moreover, in multi-cloud application management platforms such as Right Scale or Bitnami service requesters have the option to deploy parts of their applications on different clouds. Service matching research aims at finding suitable services according to the service requester's requirements. These requirements cover a wide range of properties such as functional, quality of service (QoS) and compliance.

The service matching problem is discussed in the literature in different contexts: Internet of Services, SOA, Semantic Web Services and Cloud Services. For service descriptions, several languages are suggested, each focusing on another group of service properties depending on the context such as WSDL [3], Linked-USDL1] [4], SMI] [5] and OWL-S3] [6]. Based on the context, the scope of matching covers QoS matching, inputoutput matching, precondition and postcondition matching.

Due to different opinions on the content of the service descriptions and different contexts, a variety of service matching approaches have been suggested. However, these approaches have different problem definitions and have to be examined in detail in order to identify the subproblems solved in each.

\footnotetext{
${ }^{1}$ Linked Unified Service Description Language

${ }^{2}$ Service Measurement Index

${ }^{3}$ Semantic Markup for Web Services
}

Research questions that this survey attempts to answer are:

Q1 How do they select QoS parameters to work with? Based on interviews, non-functional properties research or datasets?

Q2 Where do the service descriptions stem from: cloud computing or web services context?

Q3 What context do they target in their problem definitions? Web services, semantic web services or cloud computing in particular?

Q4 Are there any approaches which meet the essential requirements of cloud service marketplaces?

Q5 Do the existing approaches support all data types to be matched?

Our survey builds on the related work presented in [7] and [8]. These surveys provide a good overview of service matching approaches, however they have different focuses, onthe-fly computing and cloud service composition, respectively.

To answer our research questions Q1, Q2, and Q3 we examine the service description languages used, the source of service descriptions and the research or project context where the matching approach was developed. For Q4 and Q5, we assess to what extent the service matching approaches accomplish their objectives with an in-depth examination of the most relevant approaches for the context where the user takes the final decision of service selection. Moreover, we provide clear statements on the relation between the objectives and the service matching context.

This paper is organized as follows: The next section presents the survey procedure. Section 3 examines the existing approaches to answer the research questions. Section 4 presents the discussion.

\section{SURVEY PROCEDURE}

Within ACM, IEEE, and Springer digital libraries, we have searched with the keywords "service composition", "service matching", "service matchmaking", "service selection", and "QoS". Following the references, we added some more to the list. We omitted the approaches which do not fully explain how to perform QoS matching or provide only initial steps. Following this, we evaluated the approaches with two essential requirements in the cloud service marketplaces context: service requester perspective and handling incomplete knowledge. Section III examines the related work to answer the research questions Q1-Q5. 


\section{Existing Service Matching ApProaches}

As service matchmaking approaches evolve, several methods and toolsets are suggested and evaluated in the literature. This section examines existing approaches closely.

Table I shows the examination of matching approaches to answer the research questions provided above. Each column is described as follows:

Service Descriptions Source lists the service description language used for describing the services or the source for the QoS parameters in the service descriptions. This information is utilized to answer Q1 and Q2.

Target Context explains for which context the matching approach was designed, since the research objectives might vary accordingly. Moreover, the target features of the matcher depend on the analysis of the QoS properties to be matched. This field targets answering Q3.

Calculation concepts refer to the mathematical model(s) used to structure the problem 4

Three main sources for the service descriptions are: i) Numerical QoS parameters, non-functional properties research by Al-Masri and Mahmoud [9] and by Menasce et al. [10] in web services context, examples include execution time, price, availability, ii) WSDL input and output parameters, iii) Semantic web service challenge and OWL-S. The specific requirements of service matchmaking in cloud computing is to be further investigated. Apart from the contributions by the developers of USDL and Linked USDL [4], and SMI [11] themselves, there are no matching approaches which are applied to these cloud context non-functional properties.

Table $\amalg$ aims at answering Q4. It evaluates the approaches with two essential requirements in the cloud service marketplaces context. Non-technical service requester perspective assesses if the matcher answers one or all the needs of non-technical requesters with QoS requirements by allowing them to: i) make the final decision, ii) set weights on each constraint, iii) specify constraints for each property which will be compared to the service specifications rather than arranging the repository in itself, iv) specify fuzzy requests. Incomplete knowledge refers to how the matchmaking method deals with missing constraints in the service query and missing properties in the service description.

In the list provided in the survey by Platenius et al. [7], the QoS matching approaches which explicitly deal with incomplete knowledge are: [18], [25], and [26]. Some approaches provide gradual results which implicitly deal with it: [19], [27], and [28].

Table III aims at answering Q5. Some approaches only explain how the format of the constraint should be, however they do not state how the service descriptions should be.

${ }^{4} \mathrm{FL}=$ Fuzzy Logic $\mathrm{MIP}=$ Mixed-Integer Programming $\mathrm{CP}=\mathrm{Constraint}$ Programming MMKP,MCOP=Multidimensional Multiple-choice 0-1 Knapsack Problem, Multi-Constrained Optimal Path FL=Fuzzy Logic AHP=Analytic Hierarchy Process NFP=Non-Functional Property IFS=Intuitionistic Fuzzy Sets IOPE= Input, Output, Precondition, Effect FL=Fuzzy Logic FST=Fuzzy Set Theory SR=Subsumption Reasoning PCM=Policy Centered Meta-Model SWRL=Semantic Web Rule Language IFS=Intuitionistic Fuzzy Sets SWS= Semantic Web Services
Mostly, the QoS property is described as a tuple that can be added to existing service description languages.

The work by Elevan and Zhao [22] states that nonfunctional properties should be added to the service matching process, however they do not specify what they improve from other matching approaches that handle non-functional properties. Moreover, they assume the service descriptions to be equal to the query in terms of properties they include.

The aim of Wang [25] is to develop a matchmaking approach for QoS properties which cannot be measured and depend largely on the perception of service providers and consumers. It models the problem as a fuzzy multi-criteria decision making problem. The proposed method takes linguistic terms which express the decision maker's opinion on the QoS property as input; e.g., very good, fair, poor. These linguistic terms are converted to intuitionistic fuzzy numbers, and scores for each service based on decision maker's weighting for each property are calculated. Finally, the results of the decision makers are aggregated, and the services are ordered from the best to the worst.

Mobedpour and Ding [23] state that in existing matching approaches such as [14] and [30] that the non-technical or inexperienced service requesters are expected to learn their service query languages or to gain background knowledge on defining fuzzy membership functions. To solve this problem, the authors propose a process where the service requester is supported and guided by the system to formulate QoS queries along with an improved selection model. In contrast to the approach by Wang [25], the authors utilize interval clustering to classify services under the linguistic terms, which also decreases user effort. These ideas are implemented in a prototype which uses the algorithm presented in [14] as a baseline. The experiments show that more accurate results are achieved while the response time remains as before. However, no user reviews are conducted to evaluate the main purpose of this approach. Service descriptions do not contain any enumeration parameters and parameters related to business. This results in that the system does not support a use case where there are different price models for several editions of a service.

Mobedpour and Ding [23] criticize fuzzy models approaches since they require high user evaluation effort (when they have to define fuzzy numbers or membership functions) and that they might be wrong since they are based on personal opinions of the decision makers. Moreover, they do not consider crisp data. However, the approach represented in [25] takes linguistic terms as input which does not require substantial user effort. On the other hand, the QoS description is based on users' personal impressions. The last step of aggregating the scores to rank the services improves this aspect to some extent.

Bacciu et al. [26] address the service composition problem in two steps: integration of heterogeneous services and dynamic service selection-since the service properties change due to resource availability or network connectivity variations. The authors present three artifacts: a method for fuzzy specification of QoS parameters both in service requests and descriptions, a matchmaking procedure for those, and a method which targets the dynamic update of service descriptions. For 


\begin{tabular}{|c|c|c|c|}
\hline Research Work & Properties & & \\
\hline Matcher & Service Descriptions Source & Target Context & Calculation Concepts \\
\hline Yu et al. [12] & $\begin{array}{l}\text { numeric QoS parameters, cites non- } \\
\text { functional properties research in web } \\
\text { services context with focus on per- } \\
\text { formance and cost optimization by } \\
\text { Menasce }\end{array}$ & web services composition in SOA & MMKP, MCOP \\
\hline Hu et al. [13] & numeric QoS parameters by Menasce & web services composition in SOA & vector normalization \\
\hline Kritikos and Plexousakis 14 & $\begin{array}{l}\text { numeric QoS parameters along with } \\
\text { their measurement units and methods in } \\
\text { OWL-Q }[15] \text {, OWL-S }\end{array}$ & WS & MIP \\
\hline Kritikos and Plexousakis [16] & OWL-Q 15], OWL-S & WS & $\mathrm{CP}$ \\
\hline Jie et al. [17] & SWRL rules, OWL-S, WSDL & semantic WS, sws-challenge.org & - \\
\hline Palmonari et al. [18] & WSMO, WSML & semantic WS & - \\
\hline D’Mello et al. [19] & OWL-S & semantic WS in IoS, e-shopping domain & SR \\
\hline Sarang Sukumar et al. 20] & WSDL input output parameters & WS from IBM UDDI registries & Peano space filling curve \\
\hline Eleyan and Zhao [21] & as described in [22] & WS & $\begin{array}{l}\text { AHP and } \\
\text { Euclidean distance }\end{array}$ \\
\hline Mobedpour and Ding [23] & numeric QoS with QWS dataset 9],[24] & WS & FL \\
\hline Wang 25] & user perception & service marketplace & IFS,FL \\
\hline Bacciu et al. [26] & numeric QoS parameters & service composition & FST,FL \\
\hline Liu et al. [27], [28] & WSDL + OWL-S,WSMO & semantic WS & SR, FL \\
\hline
\end{tabular}

TABLE I: Examination for Service Descriptions Used

\begin{tabular}{|c|c|c|c|}
\hline Research Work & Properties & & \\
\hline Matchmaker & Service Requester Perspective & $\begin{array}{l}\text { Incomplete Knowledge } \\
\text { (requester-provider) }\end{array}$ & Adequate \\
\hline Yu et al. [12] & no, intended for dynamic service composition & works with precise data & no \\
\hline Hu et al. [13] & yes, weights on constraints & no & no \\
\hline Kritikos and Plexousakis [14] & yes, constraints for each property & yes,classified results & yes \\
\hline Kritikos and Plexousakis [16] & yes, classifies according to requester's needs & $\begin{array}{l}\text { implicitly yes, two of the algorithms return clas- } \\
\text { sified results }\end{array}$ & yes \\
\hline Jie et al. [17] & performs comparison of requesters constraints to services & not explicitly documented & no \\
\hline Palmonari et al. [18] & $\begin{array}{l}\text { yes, but how QoS request will be converted WS-Policy or } \\
\text { WSLA and to PCM is not documented }\end{array}$ & both on the requester and the provider side & no \\
\hline D'Mello et al. 19] & $\begin{array}{l}\text { merely ordering service descriptions, no comparison to ser- } \\
\text { vice requester's constraints }\end{array}$ & $\begin{array}{l}\text { implicitly yes, returns gradual results using seman- } \\
\text { tic distances }\end{array}$ & no \\
\hline Sarang Sukumar et al. [20] & no, clusters services with similar ones & not explicitly documented & no \\
\hline Elevan and Zhao [21] & $\begin{array}{l}\text { yes, uses AHP }{ }^{a} \text { for converting the service requesters pref- } \\
\text { erences to weights }\end{array}$ & implicitly yes,ranks services in preference order & yes \\
\hline Mobedpour and Ding 23] & yes, user-centered & $\begin{array}{l}\text { implicitly yes, returns super-exact and partial } \\
\text { matches }\end{array}$ & yes \\
\hline Wang [25] & yes, addresses imprecise preferences of service requesters & on the provider side & yes \\
\hline Bacciu et al. [26] & yes, fuzzy sets to allow imprecise service requests & on the provider side & yes \\
\hline Liu et al. [27], [28] & yes, constraints for each property & gradual results & yes \\
\hline
\end{tabular}

${ }^{a}$ Analytic Hierarchy Process

TABLE II: Evaluation for Essential Requirements of Cloud Service Marketplaces

\begin{tabular}{|c|c|c|c|c|}
\hline Research Work & Properties & & & \\
\hline Matchmaker & $\begin{array}{l}\text { QoS Constraint } \\
\text { Description }\end{array}$ & $\begin{array}{l}\text { Service } \\
\text { Description }\end{array}$ & Data Types of Constraints & Prototype \\
\hline [21] & as described in [22] & WSDL extension & numeric & In C\# \\
\hline 23] & $\begin{array}{l}\text { QoS query language which sep- } \\
\text { arates relaxation and preference } \\
\text { orders [29] }\end{array}$ & QWS dataset[9], [24] & $\begin{array}{l}\text { fuzzy } \llbracket \text {, range, discrete nu- } \\
\text { meric data }\end{array}$ & $\begin{array}{l}\text { non-functional matching layer: C\#, } \\
\text { ASP.NET, lp_solve }{ }^{D}\end{array}$ \\
\hline [26] & Fuzzy Sets & $\begin{array}{l}\text { Trapezoidal fuzzy num- } \\
\text { bers }\end{array}$ & Only Numeric Examples & $\begin{array}{l}\text { Extension in MATLAB, Dino Bro- } \\
\text { ker in Java }\end{array}$ \\
\hline [14], 16] & as described in [15] & as described in [15] & numeric, range & using MATLAB and Java \\
\hline
\end{tabular}

${ }^{a}$ only for numeric properties such as reliability, response time

${ }^{b}$ functional matching layer: Lucene and Java

${ }^{c}$ this one addresses non-measurable QoS properties which the author calls non-functional properties such as reliability, integrity,availability and security

TABLE III: Summary of the Most Relevant Related Work 
doing so, they extend an existing service broker which had crisp matching functionality with the imprecise QoS parameter support. Bacciu et al. [26] report that the extension in MATLAB code is planned to be integrated to Dino Broker [31], however, there are no publications about the integration. The available version of Dino Broker is in Java and the published code does not contain the fuzzy extension. Bacciu et al. [26] present an example of variational scope as a source of fuzziness. This approach expresses numeric QoS descriptions and requirements as trapezoidal fuzzy numbers. The authors assume that when a QoS requirement is a range such as "availability between $95 \%$ and $99 \%$ ", the acceptable service specifications start at $94 \%$ with a lower membership value, increasing to the best membership value between $96 \%$ and $98 \%$, and the membership value starts decreasing at $98 \%$ and ends at $100 \%$. The idea that neighbor values of the specified range are also likely acceptable by the service requester is correct on the lower side, however on the upper side it does not make sense for properties with positive tendency because it considers $99 \%$ less good than $98 \%$, although availability is a high-value preferred property. The authors give examples for the application with numeric values, however not with enumeration type QoS descriptions such as location or compatible browsers, although it is mentioned that the operators of set theory can be generalized to fuzzy set theory.

\section{DISCUSSION}

At first glance, there are cloud service matchmakers which try to solve the same service matchmaking problem. However, the problem definition varies according to the use cases and project contexts. For this reason, they either optimize on automation and leave the usability issues aside or vice versa. The different problem definitions result in their solutions not being directly comparable.

From our analysis in Table \, we conclude the following. In most cases, the project context provides the service description language used. If the service description language is an ontology, the service matchmaker is based on ontology reasoners. In other cases, the service matchmakers use different mathematical methods. However, the service matchmakers vary also because of other factors: the target service requesters and their definition for the process of service matchmaking.

One group is for automatic service composition, where the requester is another service and the service matchmaker has to find the best service specification for the request. Another group is user-oriented where the requester is a person who makes the final service selection from a list of alternative services. For the former group, the process of service matchmaking ends with the presentation of the optimal solution to the problem. For the latter group, further requirements are considered: (i) a categorization of results as very good, good and acceptable, (ii) providing an initial list and then applying relaxation, (iii) step-by-step guidance for the service requester to add constraints to the request. Another problem definition aims at answering "a developer's query" which focuses on matching the input output parameters of functions. An additional consideration in the related work is static and dynamic service descriptions, some approaches foresee an integrated monitoring module and updating the service descriptions accordingly (Q3).
Table II shows that there are some approaches which meet the essential requirements of cloud service marketplaces on a high level (Q4).

The service description languages mostly provide very generic property definitions, therefore the constructs give too much freedom to the person who describes the service and no standardized measures for most of the properties. While service description languages such as Linked USDL [4] and Service Measurement Index [5] define properties of services in very detailed yet generic ways, most existing service matchers use only numerical values as examples for their proof-ofconcept implementations.

In the existing approaches, the service descriptions stem mostly from the web services context. Some QoS properties which are specific to cloud services are not considered, for example scalability, elasticity and different price models. Moreover, some matching approaches do not provide concrete examples for the service properties their service matcher targets. An example for this is the n-ary constraints in the mixed integer programming approach [14]. Another example is the constraint-specification combinations with intervals and values. While they examine interval to interval exact matching in great detail (this combination comes quite rarely in our SDL and SLA parameters in general), they do not solve the feature list matching problem $(\mathbf{Q 1 , Q 2})$.

In our work [32], we provide an analysis of QoS properties in service descriptions. Based on this analysis, we define the subproblems of service matching as discrete numeric matching, enumeration matching, feature list matching, interval matching, and fuzziness. We examine the matching approaches presented in [23], [26] and [31], the three complementary papers [14],[16], and [33], and the approach presented in [25]. Most of the service matchers address discrete numeric matching and interval matching. Some matchers consider also enumerations: the query and the specification can take only one of the values from a predefined list. In feature list matching, both the query and the specification can take multiple values from a predefined list and a greater overlap of these two lists is targeted. However, none of the service matchers cover this subproblem (Q5).

\section{CONClusion AND Future Work}

The differences in the definition of the service matching problem results in the authors defining the functional requirements for the service matcher. The tools are selected according to the requirements. The different problem definitions of the related work result in conceptual artifacts which should be carefully examined for reusing. A catalog of components which address the subproblems of service matching can be useful to identify building blocks available to be combined.

\section{ACKNOWLEDGMENT}

This work is supported by the Horizon 2020 EU funded Integrated project CYCLONE5, grant number 644925.

\footnotetext{
${ }^{5}$ cyclone-project.eu
} 


\section{REFERENCES}

[1] Google. (2014, Oct.) Google Apps Marketplace. [Online]. Available: https://www.google.com/enterprise/marketplace/

[2] Salesforce. (2014, Oct.) App Exchange. [Online]. Available: https://appexchange.salesforce.com/

[3] E. Christensen, F. Curbera, G. Meredith, S. Weerawarana et al., "Web services description language (WSDL) 1.1," 2001.

[4] C. Pedrinaci, J. Cardoso, and T. Leidig. (2009, Jun.) Linked USDL. [Online]. Available: https://github.com/linked-usdl

[5] C. M. U. CSMIC. Cloud Services Measures for Global Use: The Service Measurement Index (SMI). [Online]. Available: http://csmic.org/resources/

[6] D. Martin, M. Burstein, J. Hobbs, O. Lassila, D. McDermott, S. McIlraith, S. Narayanan, M. Paolucci, B. Parsia, T. Payne et al., "OWL-S: Semantic Markup For Web Services," W3C Member Submission, vol. 22, pp. 2007-04, 2004.

[7] M. C. Platenius, M. von Detten, S. Becker, W. Schäfer, and G. Engels, "A Survey of Fuzzy Service Matching Approaches In the Context of On-The-Fly Computing," in Proceedings of the 16th International ACM Sigsoft Symposium on Component-Based Software Engineering. ACM, 2013, pp. 143-152.

[8] A. Jula, E. Sundararajan, and Z. Othman, "Cloud Computing Service Composition: A Systematic Literature Review," Expert Systems with Applications, vol. 41, no. 8, pp. 3809-3824, 2014.

[9] E. Al-Masri and Q. H. Mahmoud, "QoS-Based Discovery and Ranking of Web Services," in Proceedings of 16th International Conference on Computer Communications and Networks, 2007. ICCCN 2007. IEEE, 2007, pp. 529-534.

[10] D. Menasce et al., "Composing Web Services: A QoS View," Internet Computing, IEEE, vol. 8, no. 6, pp. 88-90, 2004.

[11] S. K. Garg, S. Versteeg, and R. Buyya, "A Framework for Ranking of Cloud Computing Services," Future Generation Computer Systems, vol. 29, no. 4, pp. 1012-1023, 2013.

[12] T. Yu, Y. Zhang, and K.-J. Lin, "Efficient Algorithms for Web Services Selection with End-to-End QoS Constraints," ACM Transactions on the Web (TWEB), vol. 1, no. 1, p. 6, 2007.

[13] R. Hu, J.-x. Liu, Z.-h. Liao, and J. Liu, "A Web Service Matchmaking Algorithm Based on an Extended QoS Model," in IEEE International Conference on Networking, Sensing and Control, 2008. ICNSC 2008. IEEE, 2008, pp. 1565-1570.

[14] K. Kritikos and D. Plexousakis, "Mixed-Integer Programming for QoS-Based Web Service Matchmaking," IEEE Trans. Serv. Comput., vol. 2, no. 2, pp. 122-139, Apr. 2009. [Online]. Available: http://dx.doi.org/10.1109/TSC.2009.10

[15] K. Kritikos and D. Plexousakis, "Semantic qos metric matching," in Proceedings of the European Conference on Web Services, ser. ECOWS '06. Washington, DC, USA: IEEE Computer Society, 2006, pp. 265274. [Online]. Available: http://dx.doi.org/10.1109/ECOWS.2006.34

[16] K. Kritikos and D. Plexousakis, "Semantic QoS-Based Web Service Discovery Algorithms," in Proceedings of the Fifth European Conference on Web Services, ser. ECOWS '07. Washington, DC, USA: IEEE Computer Society, 2007, pp. 181-190. [Online]. Available: http://dx.doi.org/10.1109/ECOWS.2007.21

[17] L. Y. Jie, R. Kanagasabai et al., "Dynamic Discovery of Complex Constraint-Based Semantic Web Services," in Fifth IEEE International Conference on Semantic Computing (ICSC), 2011. IEEE, 2011, pp. 51-58.

[18] M. Palmonari, M. Comerio, and F. De Paoli, "Effective and Flexible NFP-Based Ranking of Web Services," in Service-Oriented Computing. Springer, 2009, pp. 546-560.

[19] D. A. D'Mello, I. Kaur, N. Ram, and V. Ananthanarayana, "Semantic Web Service Selection Based On Business Offering," in Proceedings of the Second UKSIM European Symposium on Computer Modeling and Simulation, 2008. EMS'08. IEEE, 2008, pp. 476-481.

[20] A. Sarang Sukumar, J. Loganathan, and T. Geetha, "Clustering Web Services Based on Multi-Criteria Service Dominance Relationship Using Peano Space Filling Curve," in International Conference on Data Science \& Engineering (ICDSE), 2012. IEEE, 2012, pp. 13-18.

[21] A. Eleyan and L. Zhao, "Service Selection Using Quality Matchmaking," in International Conference on Communications and Information Technology (ICCIT), 2011. IEEE, 2011, pp. 107-115.

[22] A. Eleyan and L. Zhao, "Extending WSDL and UUDI with Quality Service Selection Criteria," in Proceedings of the 3rd International Symposium on Web Services, 2010.

[23] D. Mobedpour and C. Ding, "User-centered Design of a QoS-based Web
Service Selection System," Service Oriented Computing and Applications, pp. 1-11, 2013.

[24] E. Al-Masri and Q. H. Mahmoud, "Discovering the best web service," in Proceedings of the 16th International Conference on World Wide Web. ACM, 2007, pp. 1257-1258.

[25] P. Wang, "QoS-Aware Web Services Selection with Intuitionistic Fuzzy Set Under Consumers Vague Perception," Expert Systems with Applications, vol. 36, no. 3, pp. 4460-4466, 2009.

[26] D. Bacciu, M. G. Buscemi, and L. Mkrtchyan, "Adaptive Fuzzy-Valued Service Selection," in Proceedings of the 2010 ACM Symposium on Applied Computing. ACM, 2010, pp. 2467-2471.

[27] M. Liu, W. Shen, Q. Hao, and J. Yan, "An Weighted Ontology-Based Semantic Similarity Algorithm for Web Service," Expert Syst. Appl., vol. 36, no. 10, pp. 12480-12490, Dec. 2009. [Online]. Available: http://dx.doi.org/10.1016/j.eswa.2009.04.034

[28] M. Liu, W. Shen, Q. Hao, J. Yan, and L. Bai, "A Fuzzy Matchmaking Approach for Semantic Web Services with Application to Collaborative Material Selection," Computers in Industry, vol. 63, no. 3, pp. 193-209, 2012.

[29] D. Mobedpour, C. Ding, and C.-H. Chi, "A QoS Query Language for User-Centric Web Service Selection," in IEEE International Conference on Services Computing (SCC), 2010, July 2010, pp. 273-280.

[30] A. Ruiz-Cortés, O. Martín-Díaz, A. Durán, and M. Toro, "Improving the Automatic Procurement of Web Services Using Constraint Programming," International Journal of Cooperative Information Systems, vol. 14, no. 04, pp. 439-467, 2005.

[31] A. Mukhija, A. Dingwall-Smith, and D. S. Rosenblum, "QoS-Aware Service Composition in Dino," in Fifth European Conference on Web Services, 2007. ECOWS'07. IEEE, 2007, pp. 3-12.

[32] B. I. Zilci, M. Slawik, and A. Küpper, "Cloud Service Matchmaking using Constraint Programming," in Proceedings of the 24th IEEE International Conference on Enabling Technologies: Infrastructure for Collaborative Enterprises. IEEE, 2015.

[33] K. Kritikos and D. Plexousakis, "Evaluation of QoS-Based Web Service Matchmaking Algorithms," in Proceedings of the 2008 IEEE Congress on Services - Part I, ser. SERVICES '08. Washington, DC, USA: IEEE Computer Society, 2008, pp. 567-574. 